\title{
Database Development and Statistical Analysis of Survival in a Clinical and Historical Coort of Dogs Affected by Myxomatous Mitral Valve Disease Treated With Different Therapeutic Protocols Using Causal Inference Techniques
}

Mara Bagardi ( $\nabla$ mara.bagardi@unimi.it)

Universita degli Studi di Milano Facolta di Medicina Veterinaria https://orcid.org/0000-0002-52473796

\section{Valentina Palermo}

Anderson Moores Veterinary Specialists

Chiara Locatelli

Universita degli Studi di Milano Facolta di Medicina Veterinaria

Fabio Maria Colombo

Universita degli Studi di Milano Facolta di Medicina Veterinaria

Laura Pazzagli

Karolinska Institutets folkhälsoakademi: Karolinska Institutet

Paola Giuseppina Brambilla

Universita degli Studi di Milano Facolta di Medicina Veterinaria

\section{Research Article}

Keywords: dog, myxomatous mitral valve disease, survival analysis, causal inference techniques

Posted Date: August 17th, 2021

DOI: https://doi.org/10.21203/rs.3.rs-794366/v1

License: (c) (i) This work is licensed under a Creative Commons Attribution 4.0 International License.

Read Full License 


\section{Abstract}

The aim of this work was to retrospectively evaluate the influence of different therapeutic protocols (loop diuretics, ACE-inhibitors, spironolactone +/- pimobendan) on the survival time (ST) of dogs with myxomatous mitral valve disease at different stages using an Inverse Probability Weighting (IPW) analysis.

An IPW method was used to minimize confounding and IPW weighted time-repeated logistic model was used to approximate survival curves (SCs) and calculate survival differences. Subjects were allocated into cases (CA) and controls (CO). Dogs in American College of Veterinary Internal Medicine (ACVIM) B2 class treated with pimobendan (+/- ACE-inhibitors) were selected for the CA group, as well as symptomatic patients (ACVIM class $\mathrm{C}$ ) threated with triple (furosemide, ACE-inhibitor, pimobendan) or quadruple (furosemide, ACE-inhibitor, pimobendan and spironolactone) therapy. The CO group included ACVIM class B2 dogs not treated with any medication and ACVIM C dogs treated with a combination of furosemide and ACE-inhibitor/spironolactone without pimobendan.

The SC of the CA group crossed the CO group at 1634 days. The difference between the two SCs at the time of maximum survival difference in favour of the $\mathrm{CO}$ group was $11.3 \%(\mathrm{Cl} 1.7 \%-20.9 \%)$ (significant), in favour of the $\mathrm{CA}$ group was $3.9 \%(\mathrm{Cl}-8.6 \%-16.4 \%)$ (not significant) and at the mean ST was $3.6 \%$ (Cl $-8.5 \%-15.7 \%$ ) (not significant) in favour of the CA group. For times greater than 1634 days the survival was in favour of the CA group, there were no statistically significant differences in survival in favour of the CA group in this clinical population.

\section{Introduction}

The cardiovascular activity of pimobendan is known since 1987 and from that time many studies have been performed to define its effects on the survival time with different severity of heart diseases in dogs (Boyle \& Leeche 2012, Boswood et al. 2016, Häggström et al. 2008, Imagawa et al. 1987, Sumerfield et al. 2012). Overall, pimobendan has shown a positive association with longer survival in dogs affected by myxomatous mitral valve disease (MMVD) and dilatative cardiomyopathy and different studies applied different statistical approaches.

The multicentric blinded, randomized, placebo or positive (benazepril) controlled clinical trials with the highest scientific evidence were focused on patients affected by MMVD prospectively recruited, considering the inclusion and exclusion criteria defined before (Boswood et al. 2016, Häggström et al. 2008).

The onset of congestive heart failure (CHF), cardiac-related death or euthanasia were considered the primary and/or secondary endpoint of these studies (Boswood et al. 2016, Häggström et al. 2008). The Kaplan Mayer method was used to calculate the median survival time to endpoint of the enrolled dogs and a log-rank test determined whether a significant difference existed between the treatment groups (Boswood et al. 2016, Häggström et al. 2008). The multivariable Cox proportional hazard analysis was 
performed to test the prognostic significance of the clinical and demographic parameters recorded in the different therapeutic groups.

The confounding bias that may affect retrospective studies, often influences the evaluation of the differences in survival times (ST) between treatment groups and the association of the variables of interest with the survival time. In these circumstances a statistical approach coming from the causal inference literature could be applied to determine whether this method leads to results consistent with findings from randomized studies, also in retrospective data analyses. Moreover, populations included in randomized studies may be very selected and observational data related to populations of patients treated in clinical practice may complement evidence from trials and contribute to generate new evidence on treatment effects.

Among the causal inference methods, the Inverse Probability Weighting (IPW) method uses two regression models, one for the exposure and one for the outcome. The regression model for the exposure is referred to a propensity score (PS) model and estimates the probability of being exposed to the treatment of interest conditional on confounding factors for all subjects in the study population. The model for the exposure is used to construct weights which will be assigned to the subjects in the study population. The second regression model is the outcome model, representing the main analysis model, which aims to estimate the association of the exposures of interest with the outcome variable. The IPW method aims to minimize confounding bias by improving exchangeability between exposed and unexposed subjects with the final aim to emulate the randomization of the treatment. To reach this aim subjects with lower probability of being exposed will receive a bigger weight to avoid underrepresentation of this part of the population and vice versa subjects with higher probability of being exposed will receive a lower weight. Adjusting for confounding and reproducing a pseudo population where each subject should be potentially exposed and not exposed to the treatment of interest, the IPW method aims to reach a causal interpretation of the findings under the assumption of no unmeasured confounding and other important assumptions implied by causal inference methods.

An IPW method was used (Cole \& Hernán 2008) to estimate the association between the exposure to the type of therapeutic treatment (including or not pimobendan) and death by cardiac or other causes. Specifically, the aim of this study was to evaluate the influence of different therapeutic protocols, including loop diuretics (furosemide), ACE-inhibitors, and spironolactone with or without the inodilator pimobendan, on the ST of dogs affected by different stages of MMVD (asymptomatic with cardiac remodelling and symptomatic) via an IPW analysis. To the best of our knowledge this statistical technique has never been used for this type of retrospective study in veterinary medicine.

\section{Material And Methods}

- Medical records (2000-2020) of client-owned dogs referred to the Cardiology Unit of the Department of Veterinary Medicine of the University of Milan, were retrospectively reviewed. 
Owner consent was routinely requested before the first examination of each dog.

From the beginning of the study to 2009, the admitted dogs were classified according to the International Small Animal Cardiac Health Council (ISACHC) classes (Woodbridge 1994) and from 2010 to 2020 according to the American College of Veterinary Internal Medicine classification (ACVIM) (Keene et al. 2019). To compare the ST of the subjects, a univocal classification was needed, and patients classified in ISACHC classes II, IIla and IIIb were reallocated to ACVIM class C.

The clinical records were selected according to the following inclusion criteria: complete clinical findings including signalment, history (increased resting respiratory rate, cough, dyspnea) physical examination, thoracic radiographs, and electrocardiogram (ECG).

The diagnosis of MMVD and the ACVIM classification as B2 and C were based on echocardiographic and Doppler evaluation executed by veterinarians focused on specialist cardiology practice and in accordance with the recommendations of the Echocardiography Committee of the Specialty of Cardiology (Thomas et al. 1993, Hansson et al. 2002, Keene et al. 2019). The echocardiographic parameters considered for the statistical analysis were the left atrial to aortic root ratio (LA/Ao), the left ventricular end diastolic and end systolic volumes indexed for body surface area (EDVI and ESVI), the left ventricular internal diameters in diastole and systole normalized for body weight (LVIDdN and LVIDsN) and the left ventricular fractional shortening (FS\%) (Cornell et al. 2004, Teichholz et al. 1976). The trans-mitral flow (E wave peak velocity, A wave peak velocity, E/A ratio) was measured using pulsed-wave Doppler (PWD) from the left fourchamber apical view.

The presence and the severity of pulmonary hypertension $(\mathrm{PH})$ were based on the tricuspid regurgitation velocity (TRV). The PH was classified as low, moderate, or severe as reported in the literature (Acierno et al. 2018).

Clinical records of subjects affected by any other heart disease apart from MMVD, clinically significant systemic disease, or other significant organ dysfunction were excluded. Additionally, subjects with primary hypertension were also excluded from the study (Acierno et al. 2018).

The subjects were then categorized into "case" group (CA) and "control" group (CO).

Dogs belonging to class $\mathrm{B} 2$, treated with pimobendan alone or in combination with ACE-inhibitors were included in the CA group, as well as the symptomatic patients (ACVIM class C) threated with triple (furosemide, ACE-inhibitor, pimobendan) or quadruple (furosemide, ACE-inhibitor, pimobendan and spironolactone) therapy. The CO group included untreated ACVIM B2 dogs and ACVIM C patients treated with a combination of furosemide, ACE-inhibitor +/-spironolactone, without pimobendan.

Endpoint of the study was the animal's death (cardiac-related death or euthanasia, and extracardiac causes). Owners were contacted by phone to complete medical records' data and to collect information on subjects' follow up. Dogs lost at follow-up were not included. 
Data obtained from clinical records from 2000 to 2020 were then statistically analysed.

\section{Statistical analysis}

Initially, relevant, or confounding variables to be used in the IPW analysis were dichotomized to 1 (higher risk) or 0 (lower risk) levels, according to the risk threshold derived from the literature for each of the covariates. The variables considered to be influential exclusively on MST were age, cough, dyspnea, and ejection fraction (EF). The confounding variables that affected both survival and treatment assignment were EDVI, LA/Ao ratio, and the presence of $\mathrm{PH}$. Other unmeasured confounding variables for all subjects were LVIDdN and LVIDsN (Borgarelli et al. 2012, Borgarelli et al. 2015, Kim et al. 2017, Madeleine et al. 2018).

Survival curves were estimated, both for dogs exposed (CA) and unexposed (CO) to pimobendan treatment, by a IPW weighted model following the method described by Hernán and Robins (Hernán \& Robins 2020). Firstly, weights were derived as "stabilized inverse probability weights" (SIPW), i.e., the ratio between the marginal probability density of the exposure and the probability density of the exposure conditional to covariates such as age, pulmonary hypertension, cough, dyspnoea, EDVI, LA/Ao and EF\% (Hernán et al. 2001). Survival curves were estimated approximating them with a time-repeated logistic model weighted with the IPW weights and with live/dead as binary outcome. Finally, differences in survival curves among CA and CO groups were estimated using the IPW weighted logistic model and 95\% confidence intervals (Cls) were derived based on 100 bootstrap samples.

All statistical procedures were performed in the " $R$ " environment ( $R$ Core Team 2014) using the following packages: "ggplot2" (Wickham 2016), "parameters" (Lüdecke et al. 2020), "splitstackshape" (Mahto 2019) and "survminer" (Kassambara et al. 2020).

\section{Results}

The population included 398 subjects affected by MMVD, 236 (59.30\%) were classified as CA and 162 (40.70\%) as CO. Two hundred and fifty-four were males (63.82\%) and 144 were females (36.18\%). One hundred and sixty-eight were small and medium size mixed breed (42.21\%); pure breeds included 25 (6.28\%) Poodles, 25 (6.28\%) Yorkshire Terriers, 22 (5.53\%) Cavalier King Charles Spaniels, 17 (4.27\%) Shih-tzu, 17 (4.27\%) Dachshunds, 13 (3.27\%) Chihuahuas, 10 (2.51\%) Maltese dogs, 10 (2.51\%) Pinscher and other breeds in lower percentage. The mean age of the included population was $11.88 \pm 3.07$ years, the mean weight was $10.97 \pm 7.83 \mathrm{~kg}$. Clinical and echocardiographic characteristics of the included population are reported respectively in Table 1 and Table 2 . 
Table 1

Clinical characteristics expressed as mean and standard deviation of the overall population (CA + $\mathrm{CO}$ ) and of cases (CA) and controls (CO).

\begin{tabular}{|llll|}
\hline & n. & Age (years) & Weight $(\mathrm{kg})$ \\
\hline $\mathrm{CA}+\mathrm{CO}$ & 398 & $11.88 \pm 3.07$ & $10.97 \pm 7.83$ \\
\hline $\mathrm{CA}$ & 236 & $11.96 \pm 3.03$ & $10.47 \pm 7.78$ \\
\hline $\mathrm{CO}$ & 162 & $11.77 \pm 3.13$ & $11.71 \pm 7.87$ \\
\hline
\end{tabular}

Table 2

Echocardiographic characteristics expressed as mean and standard deviation of the overall population $(\mathrm{CA}+\mathrm{CO})$ and of cases (CA) and controls (CO).

\begin{tabular}{|c|c|c|c|c|c|c|c|c|c|}
\hline & $\mathrm{n}$. & LA/Ao & $\begin{array}{l}\text { EDVI } \\
\left(\mathrm{ml} / \mathrm{m}^{2}\right)\end{array}$ & $\begin{array}{l}\text { ESVI } \\
\left(\mathrm{ml} / \mathrm{m}^{2}\right)\end{array}$ & LVIDdN & LVIDsN & EF \% & SF \% & $E / A$ \\
\hline $\begin{array}{l}\text { CA } \\
+\mathrm{CO}\end{array}$ & 398 & $\begin{array}{l}2.14 \pm \\
0.49\end{array}$ & $\begin{array}{l}141.38 \pm \\
60.29\end{array}$ & $\begin{array}{l}36.29 \pm \\
20.93\end{array}$ & $\begin{array}{l}1.93 \pm \\
0.48\end{array}$ & $\begin{array}{l}1.04 \pm \\
0.33\end{array}$ & $\begin{array}{l}73.34 \pm \\
10.39\end{array}$ & $\begin{array}{l}42.67 \pm \\
9.27\end{array}$ & $\begin{array}{l}1.22 \pm \\
1.16\end{array}$ \\
\hline CA & 236 & $\begin{array}{l}2.13 \pm \\
0.49\end{array}$ & $\begin{array}{l}138.82 \pm \\
60.36\end{array}$ & $\begin{array}{l}36.07 \pm \\
20.43\end{array}$ & $\begin{array}{l}1.85 \pm \\
0.39\end{array}$ & $\begin{array}{l}1.01 \pm \\
0.31\end{array}$ & $\begin{array}{l}72.72 \pm \\
10.57\end{array}$ & $\begin{array}{l}41.87 \pm \\
8.67\end{array}$ & $\begin{array}{l}1.27 \pm \\
1.21\end{array}$ \\
\hline $\mathrm{CO}$ & 162 & $\begin{array}{l}2.14 \pm \\
0.54\end{array}$ & $\begin{array}{l}144.19 \pm \\
61.08\end{array}$ & $\begin{array}{l}36.38 \pm \\
21.82\end{array}$ & $\begin{array}{l}1.97 \pm \\
0.52\end{array}$ & $\begin{array}{l}1.10 \pm \\
0.35\end{array}$ & $\begin{array}{l}73.33 \pm \\
12.98\end{array}$ & $\begin{array}{l}43.28 \pm \\
11.06\end{array}$ & $\begin{array}{l}1.24 \pm \\
1.19\end{array}$ \\
\hline \multicolumn{10}{|c|}{$\begin{array}{l}\text { Note: } L A / A o=\text { left atrial to aortic root ratio; } E D V I=\text { left ventricular end diastolic volume indexed for } \\
\text { body surface area; ESVI = left ventricular end systolic volume indexed for body surface area; LVIDdN = } \\
\text { left ventricular internal diameter in diastole normalized for body weight; } L \text { LVID SN = left ventricular } \\
\text { internal diameter in systole normalized for body weight; } E F=\text { left ventricular ejection fraction; } F S=\text { left } \\
\text { ventricular fractional shortening; } E / A=E \text { and } A \text { wave ratio. }\end{array}$} \\
\hline
\end{tabular}

The median survival time (MST) was 479 days $\left(\mathrm{IQR}_{25}-75\right.$ 224-928) for the entire population; the MST of ACVIM class B2 was 825 days ( $\mathrm{IQR}_{25}-75$ 349-1425), while the MST of ACVIM class $C$ was 420 days

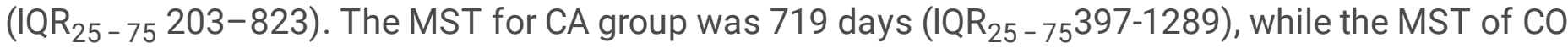
group was 698 days $\left(\mathrm{IQR}_{25-75} 362-1156\right)$.

The survival curve of the CA group crossed the survival curve of the CO group at 1634 days.

The difference between the two survival curves at the time of maximum survival difference in favour of the $\mathrm{CO}$ group was $11.3 \%(\mathrm{Cl} 1.7 \%-20.9 \%)$ (significant), while the difference at the time of maximum survival difference in favour of the CA group was $3.9 \%(\mathrm{Cl}-8.6 \%-16.4 \%)$ (not significant) and at the median survival time was $3.6 \%(\mathrm{Cl}-8.5 \%-15.7 \%)$ (not significant) in favour of the $\mathrm{CA}$ group.

The statistical analysis, carried out using the method proposed by Hernán and Robins, showed a reversal of the trend in the survival curves for CA and CO groups: in a time-frame shorter than 1634 days the $\mathrm{CO}$ group had better survival, while the CA had better survival after 1634 days (Fig. 1). 


\section{Discussion}

The results from this study are consistent with findings from a prospective, single-blinded, randomized multicenter study trial, showing that causal inference methods can be used to consolidate results from randomized trials (Wess et al. 2020).

Our study has been conducted in a clinical population including patients treated during clinical practice and shows that causal inference methods can be used to analyse data routinely collected in clinical practice to complement evidence coming from randomized trials. Data collected in clinical practice reflects treatment patterns of patients with disease severity and treatment history different from patients included in randomized trials and therefore constitutes a valuable research resource to investigate treatment effects. In clinical practice patients may be treated with different therapeutic protocols (including comedications and treatment changes) and investigating the differences in survival due to different protocols is crucial for contributing to clinical guidelines. Moreover, randomized trials may be often infeasible due to ethical and economic reasons, or they may include very selected populations. Collecting evidence on treatment effects via observational clinical data routinely collected and analysed with causal inference methods may increase awareness on treatment effectiveness and safety in more general populations encountered during clinical practice.

The IPW analysis attempted to provide a causal interpretation of the association between pimobendan and survival time, however limitations may come from the fulfillment of some of the assumptions implied by the methods such as no unmeasured confounders. In case some important confounding factors remain unmeasured the findings can still retain some residual bias. Indeed, the results of this study on the effect of pimobendan on survival may be affected by differences in disease severity of the dogs in the study population undergoing different treatment strategies. Otherwise, among the advantages of the approach is the possibility to obtain marginal estimates of the potential effect of pimobendan on survival time with respect to traditional methods which conditioning on confounding factors in the outcome model allow to have only conditional estimates. Finally, the approach is novel to veterinary medicine and other applications in the field are warranted given the increased availability of valuable clinical data routinely collected.

\section{Declarations}

Funding: not applicable.

Conflicts of interest/Competing interests: not applicable.

Availability of data and material: data is available by upon request.

Code availability: not applicable.

Author contributions 
Conceptualization and experimental design, P.G.B, V.P., M.B., F.M.C. and L.P.; methodology, V.P., M.B., F.M.C. and L.P.; data curation, V.P. and M.B.; software, F.M.C.; formal analysis, F.M.C. and M.B.; investigation, F.MC., M.B., L.P. and P.G.B.; resources, P.G.B.; writing-original draft, M.B., F.M.C., V.P., L.P. and P.G.B.; writing-review and editing, M.B., V.P., C.L., F.M.C., L.P. and P.G.B; supervision, P.G.B. and L.P; project administration, P.G.B. All authors have read and agreed to the published version of the manuscript.

Ethics approval: not applicable.

Consent to participate not applicable.

Consent for publication: not applicable.

\section{References}

1. Acierno MJ, Brown S, Coleman AE, Jepson RE, Papich M, Stepien RL, Syme HM (2018) ACVIM consensus statement: Guidelines for the identification, evaluation, and management of systemic hypertension in dogs and cats. J Vet Intern Med 32:1803-1822

2. Boyle KL, Leech E (2012) A review of the pharmacology and clinical uses of pimobendan. J Vet Emerg Crit Care (San Antonio) 22(4):398-408

3. Borgarelli M, Crosara S, Lamb K, Savarino P, La Rosa G, Tarducci A, Häggström J (2012) Survival Characteristics and Prognostic Variables of Dogs with Preclinical Chronic Degenerative Mitral Valve Disease Attributable to Myxomatous Degeneration. J Vet Intern Med 26(1):69-75

4. Borgarelli M, Abbott J, Braz-Ruivo L, Chiavegato D, Crosara S, Lamb K, Ljungvall I, Poggi M, Santilli RA, Häggström J (2015) Prevalence and prognostic importance of pulmonary hypertension in dogs with myxomatous mitral valve disease. J Vet Intern Med 29(2):569-574

5. Boswood A, Häggström J, Gordon SG, Wess G, Stepien RL, Oyama MA, Keene BW, Bonagura J, MacDonald KA, Patteson M, Smith S, Fox PR, Sanderson K, Woolley R, Szatmari V, Menaut P, Church WM, O'Sullivan ML, Jaudon J-P, Kresken J-G, Rush J, Barrett KA, Rosenthal SL, Saunders AB, Ljungvall I, Deinert M, Bomassi E, Estrada AH, Fernandez Del Palacio MJ, Moise NS, Abbott JA, Fujii Y, Spier A, Luethy MW, Santilli RA, Uechi M, Tidholm A, Watson P (2016) Effect of Pimobendan in Dogs with Preclinical Myxomatous Mitral Valve Disease and Cardiomegaly: The EPIC Study-A Randomized Clinical Trial. J Vet Intern Med 30:1765-1779

6. Cole SR, Hernán MA (2008) Constructing inverse probability weights for marginal structural models. Am J Epidemiol 168:656-664. https://doi.org/10.1093/aje/kwn164

7. Cornell CC, Kittleson MD, Della Torre P, Häggström J, Lombard CW, Pedersen HD, Vollmar A, Wey A (2004) Allometric Scaling of M-Mode Cardiac Measurements in Normal Adult Dogs. J Vet Intern Med 18:311-321

8. Häggström J, Boswood A, O'Grady M, Jons O, Smith S, Swift S, Borgarelli M, Gavaghan B, Kresken JG, Patteson M, Ablad B, Bussadori CM, Glaus T, Kovacevic A, Rapp M, Santilli RA, Tidholm A, 
Eriksson A, Belanger MC, Deinert M, Little CJ, Kvart C, French A, Ronn Landbo M, Wess G,

Eggertsdottir AV, O’Sullivan ML, Schneider M, Lombard CW, Dukes McEwan J, Willis R, Louvet A, Di

Fruscia R (2008) Effect of pimobendan or benazepril hydrochloride on survival times in dogs with

congestive heart failure caused by naturally occurring myxomatous mitral valve disease: the QUEST study. J Vet Intern Med 22:1124-1135

9. Hansson K, Häggström J, Kvart C, Lord P (2002) Left atrial to aortic root indices using twodimensional and m-mode echocardiography in cavalier King Charles spaniels with and without left atrial enlargement. Vet Radiol Ultrasound 43:568-575

10. Hernán MA, Brumback B, Robins JM (2001) Marginal Structural Models to Estimate the Joint Causal Effect of Nonrandomized Treatments. J Am Stat Assoc 96:440-448.

https://doi.org/10.1198/016214501753168154

11. Hernán MA, Robins JM (2020) Causal Inference. What If

12. Imagawa J, Satoh K, Taira N (1987) Cardiac and coronary vasodilator profile of pimobendan, a new cardiotonic drug, revealed by use of isolated, blood-perfused dog heart preparations. Heart Vessels 3(4):182-189

13. International Small Animal Cardiac Health Council (1994) Recommendations for the diagnosis and treatment of heart failure in small animals. ISACHC Publication, Woodbridge, p 5

14. Kassambara A, Kosinski M, Biecek P (2020) survminer: Drawing Survival Curves using "ggplot2\&\#8221

15. Keene BW, Atkins CE, Bonagura JD, Fox PR, Häggström J, Fuentes VL, Oyama MA, Rush JE, Stepien $\mathrm{R}$, Uechi M (2019) ACVIM consensus guidelines for the diagnosis and treatment of myxomatous mitral valve disease in dogs. J Vet Intern Med 33:1127-1140

16. Kim HT, Han SM, Song WJ, Kim B, Choi M, Yoon J, Youn HY (2017) Retrospective study of degenerative mitral valve disease in small-breed dogs: Survival and prognostic variables. J Vet Sci 18(3):369-376

17. Lüdecke D, Ben-Shachar MS, Patil I, Makowski D (2020) Extracting, Computing and Exploring the Parameters of Statistical Models using \{R\}. J Open Source Softw 5:2445.

https://doi.org/10.21105/joss.02445

18. Madeleine J, Mattin A, Boswood DB, Church DC, Brodbelt (2018) Prognostic factors in dogs with presumed degenerative mitral valve disease attending primary-care veterinary practices in the United Kingdom. J Vet Intern Med 33(2):432-444

19. Mahto A (2019) splitstackshape: Stack and Reshape. Datasets After Splitting Concatenated Values

20. R Core Team (2014) R: A Language and. Environment for Statistical Computing

21. Summerfield NJ, Boswood A, O'Grady MR, Gordon SG, Dukes-McEwan J, Oyama MA, Smith S, Patteson M, French AT, Culshaw GJ, Braz-Ruivo L, Estrada A, O'Sullivan ML, Loureiro J, Willis R, Watson $P$ (2012) Efficacy of pimobendan in the prevention of congestive heart failure or sudden death in Doberman Pinschers with preclinical dilated cardiomyopathy (the PROTECT Study). J Vet Intern Med Nov-Dec 26(6):1337-1349 
22. Teichholz LE, Kreulen T, Herman MV, Gorlin R (1976) Problems in echocardiographic volume determinations: Echocardiographic-angiographic correlations in the presence of absence of asynergy. Am J Cardiol 37:7-1

23. Thomas WP, Gaber CE, Jacobs GJ, Kaplan PM, Lombard CW, Moise NS, Moses BL (1993)

Recommendations for standards in transthoracic two-dimensional echocardiography in the dog and cat. Echocardiography Committee of the Specialty of Cardiology, American College of Veterinary Internal Medicine. J Vet Intern Med 7:247-252

24. Wess G, Kresken JG, Wendt R, Gaugele J, Killich M, Keller L, Simak J, Holler P, Bauer A, Küchenhof H, Glaus T (2020) Efficacy of adding ramipril (VAsotop) to the combination of furosemide (Lasix) and pimobendan (VEtmedin) in dogs with mitral valve degeneration: The VALVE trial. J Vet Intern Med 34(6):2232-2241

25. Wickham H (2016) ggplot2: Elegant Graphics for Data Analysis. Springer-Verlag, New York

\section{Figures}

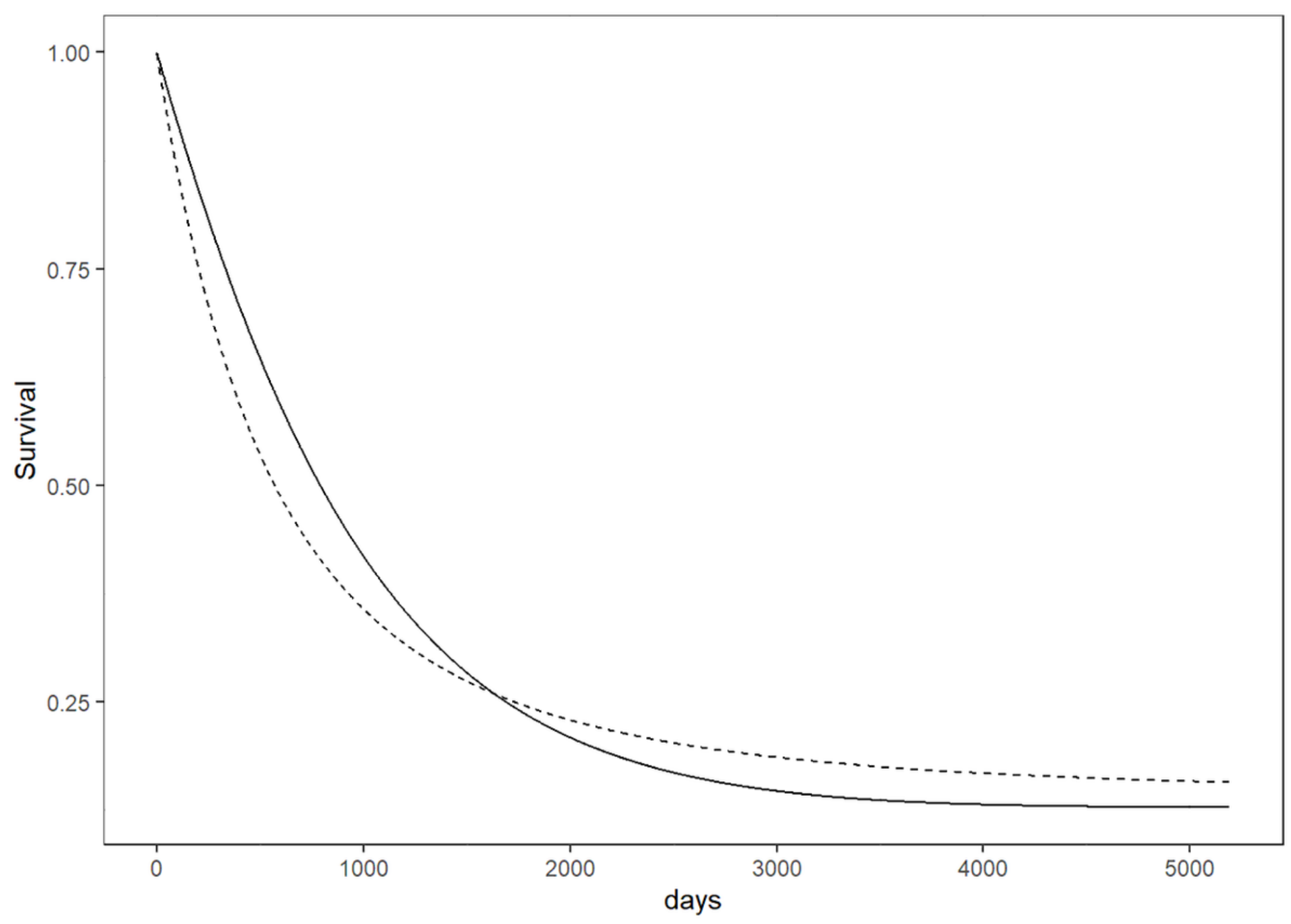

Figure 1 
Survival curves estimated via the IPW weighted time-repeated logistic model. Dashed line: Cases (CA); Solid line: Control (CO) 